\title{
Custo de Transporte em um Modelo de Crescimento Econômico com Dependência Espacial
}

\author{
João Plínio Juchem Neto \\ Universidade Federal do Pampa - Campus Alegrete \\ 97546-550, Alegrete, RS \\ E-mail: plinio@unipampa.edu.br \\ Julio Cesar Ruiz Claeyssen \\ Universidade Federal do Rio Grande do Sul - Instituto de Matemática \\ 91509-900, Porto Alegre, RS \\ E-mail: julio@mat.ufrgs.br
}

Resumo: Neste trabalho introduzimos custo de transporte no modelo de crescimento econômico espacial de Solow em uma dimensão espacial e, através de uma análise de estabilidade linear de seu ponto de equilíbrio espacialmente homogêneo, mostramos que existe um valor crítico para este custo onde o comportamento dinâmico da economia se altera, desde que a intensidade de migração da mão-de-obra seja alta o suficiente. Se o custo de transporte é maior do que este valor, o equilíbrio é estável, e a economia converge para um estado homogêneo. Caso o custo de transporte seja menor do que este valor crítico, o equilíbrio é instável e a economia desenvolve uma dinâmica espaço-temporal mais complexa, podendo haver a formação de aglomerados econômicos estáveis, de ciclos econômicos periódicos ou de ciclos aperiódicos ou caóticos, dependendo dos valores dos parâmetros utilizados.

Palavras-chave: Modelo de Crescimento Econômico, Sistema Reativo-Difusivo-Advectivo, Análise de Estabilidade Linear, Formação de Padrões Espaciais

\section{Introdução}

$\mathrm{Na}$ literatura podemos encontrar duas grandes linhas de pesquisa cujo intuito é estudar a dinâmica espaço-temporal de uma economia e, em particular, descrever como se dá o processo de aglomeração econômica. A primeira destas linhas é mais antiga (década de 1950 em diante) e advém da área de estudos denominada Ciência Regional, a qual possui um viés macroeconômico $[12,13]$, e a segunda, mais recente (década de 1990 em diante), é denominada de Nova Geografia Econômica (NGE), que trabalha com modelos microeconômicos. O modelo fundamental desta segunda linha é chamado Modelo de Centro-Periferia, e um de seus principais resultados é que o custo de transporte deve ser menor do que um determinado valor crítico para que haja formação de aglomerados econômicos $[10,11]$.

Talvez devido ao sucesso e ampla aceitação da NGE, nos anos 2000 há uma espécie de renascimento da Ciência Regional [16], com a publicação de uma série de artigos propondo e analisando modelos macroeconômicos de crescimento econômico espacial $[5,3,4,6,7,8,2]$. Matematicamente estes modelos são expressos como sistemas de equações diferenciais parciais com termos de reação e difusão, além de, em alguns trabalhos, envolver problemas de controle ótimo, quando algum tipo de análise de bem-estar está presente. 
Recentemente, em [14, 15], é proposta uma generalização do Modelo de Solow Espacial até então encontrado na literatura $[5,3,4,7,8]$, levando em consideração a interação entre as distribuições de capital e mão-de-obra (m-o). Neste trabalho é mostrado que a existência de uma migração de m-o induzida pelo capital é condição necessária para que haja a formação endógena de aglomerados e ciclos econômicos.

O presente trabalho tem por objetivo introduzir custo de transporte do produto agredado no modelo considerado em $[14,15]$ e, através de uma análise de estabilidade linear, mostrar que se este custo for suficientemente baixo, o modelo apresenta um regime de instabilitade onde pode haver a formação de aglomerados estaveis, ciclos econômicos periódicos ou aperiódicos, reproduzindo, com um modelo macroeconômico, um resultado fundamental da Nova Geografia Econômica.

\section{O Modelo}

Neste modelo consideramos um continuum de economias locais representado pelo intervalo compacto $\Omega=[0, l] \in \mathbb{R}$, onde $l>0$ é o tamanho da economia. Cada ponto $x \in \Omega$ é uma unidade produtora local possuindo uma densidade de capital $K(t, x)>0$ e de mão-de-obra $L(t, x)>0$. Estes fatores são então utilizados na produção de um bem agregado, $Y(t, x)$, através de uma função de produção de Cobb-Douglas, $Y(t, x)=A K^{\phi} L^{1-\phi}$, onde $A>0$ é o nível de tecnologia e $\phi \in(0,1)$ é a intensividade no uso do capital, ambos parâmetros constantes ao longo de toda a economia. Além disso, consideramos que a taxa de crescimento natural da mão-de-obra em cada ponto é dado por uma função logística, o que implica que esta será limitada no longo prazo.

A formulação matemática do modelo, que é derivado a partir de equações de conservação de capital e trabalho ao longo de toda a economia [14, 15], é dado pelo seguinte sistema acoplado de equações diferenciais parciais com termos de reação, difusão e advecção:

$$
\begin{aligned}
& \partial_{t} K=\left(s A K^{\phi} L^{1-\phi}-\delta K\right)+d_{K} \partial_{x x} K+\rho_{K} d_{K} \partial_{x} K \\
& \partial_{t} L=L(a-b L)+d_{L} \partial_{x x} L-\chi_{L} \partial_{x}\left(L \partial_{x} K\right) \\
& K(0, x)=K_{0}(x), L(0, x)=L_{0}(x) \\
& \partial_{x} K(t, 0)=0, \partial_{x} L(t, l)=0
\end{aligned}
$$

onde $s \in(0,1)$ é a taxa de poupança, $\delta \in(0,1)$ é a taxa de depreciação do capital, $a>0$ é a taxa de crescimento da m-o e $b>0$ é o seu coeficiente de auto-limitação; $d_{K}, d_{L}$ são os coeficientes de difusão do capital e da m-o, respectivamente (o capital/m-o tende a se difundir para regiões onde há menos capital/m-o disponível, o que respeita o princípio neoclássico do retorno marginal decrescente dos fatores, [1]); $\chi_{L}$ é o coeficiente de migração da m-o induzida pelo capital (para regiões onde há mais capital disponível); e $\rho_{K} \in(0,1)$ é o custo de transporte do produto agregado em termos físicos. O termo $\rho_{K} d_{K} \partial_{x} K$ na equação (1a) segue do pressuposto de que cada localidade paga uma fração $\sigma_{K}$ do fluxo do produto agregado passando por ela como custo de transporte (páginas 111-112 de [13]).

As distribuições iniciais de capital e m-o são dadas por (1c), e quanto às condições de contorno, consideramos que a economia é uma autarquia, ou seja, que não há transferência de capital e de m-o através de suas fronteiras. Desta forma impomos condições de contorno do tipo Neumann homogêneas (1d).

Para fins de análise, é conveniente considerar este modelo em forma adimensional. Para tanto, definimos as variáveis adimensionais:

$$
K^{*}=\frac{K}{K_{\infty}}, L^{*}=\frac{L}{L_{\infty}}, t^{*}=a t, x^{*}=\sqrt{\frac{a}{d_{K}}} x,
$$

onde $K_{\infty}=\frac{a}{b}\left(\frac{s A}{\delta}\right)^{\frac{1}{1-\phi}}$ e $L_{\infty}=\frac{a}{b}$ são os pontos de equilíbrio espacialmente homogêneos de coexistência para as distribuições de capital e mão-de-obra do modelo (1a)-(1d), respectivamente. 
Substituindo (2) em (1a)-(1d), e desconsiderando os asteriscos para manter a simplicidade notacional, obtemos o sistema em forma adimensional:

$$
\begin{aligned}
& \partial_{t} K=\beta K\left(K^{\phi-1} L^{1-\phi}-1\right)+\partial_{x x} K+\rho \partial_{x} K \\
& \partial_{t} L=L(1-L)+d \partial_{x x} L-\chi \partial_{x}\left(L \partial_{x} K\right) \\
& K(0, x)=K_{0}(x), L(0, x)=L_{0}(x) \\
& \partial_{x} K(t, 0)=0, \partial_{x} L(t, l)=0
\end{aligned}
$$

onde $\beta=\frac{\delta}{a}, d=\frac{d_{L}}{d_{K}}, \chi=\frac{a}{b} \frac{\chi_{L}}{d_{K}}\left(\frac{s A}{\delta}\right)^{\frac{1}{1-\phi}}$ e $\rho=\rho_{K} \sqrt{\frac{d_{K}}{a}}$. Note que agora os pontos de equilíbrio espacialmente homogêneos do modelo estão normalizados, isto é, $K_{\infty}=L_{\infty}=1$.

Segue de [9], com pequenas modificações, o seguinte resultado que garante que se as distribuições iniciais de capital e m-o são não-negativas então estas sempre são não-negativas, o que garante a factibilidade econômica das soluções do modelo.

Proposição 1 - Considere $K_{0}(x)$ e $L_{0}(x)$ distribuições iniciais de capital e mão-de-obra nãonegativas. Se $K(t, x)$ e $L(t, x)$ são funções $\mathcal{C}^{1}$ em relação a $t$ e $\mathcal{C}^{2}$ em relação a $x$, então as soluções de (1a)-(1d) (e também de (3a)-(3d)) são sempre não-negativas.

\section{Análise de Estabilidade}

Definindo o vetor $\mathbf{U}=(K, L)^{\top}$, podemos escrever o sistema (3a)-(3d) em forma vetorial:

$$
\begin{aligned}
& \partial_{t} \mathbf{U}=\mathbf{f}(\mathbf{U})+\mathbf{D} \partial_{x x} \mathbf{U}+\mathbf{w}(\mathbf{U}) \\
& \mathbf{U}(0, x)=\mathbf{U}_{\mathbf{0}}(x) \text { on } \Omega \\
& \partial_{x} \mathbf{U}(t, x)=\mathbf{0} \text { on } \partial \Omega
\end{aligned}
$$

onde:

$$
\mathbf{D}=\left(\begin{array}{ll}
1 & 0 \\
0 & d
\end{array}\right), \mathbf{f}(\mathbf{U})=\left(\begin{array}{c}
h \\
g
\end{array}\right)=\left(\begin{array}{c}
\beta K\left(K^{\phi-1} L^{1-\phi}-1\right) \\
L(1-L)
\end{array}\right) \text { e } \mathbf{w}(\mathbf{U})=\left(\begin{array}{c}
\rho \partial_{x} K \\
-\chi \partial_{x}\left(L \partial_{x} K\right)
\end{array}\right) .
$$

A seguir iremos analisar a estabilidade linear do único ponto de equilíbrio espacialmente homogêneo de coexistência de (4a)-(4c):

$$
\mathbf{U}_{\infty}=\left(K_{\infty}, L_{\infty}\right)^{\top}=(1,1)^{\top}
$$

seguindo $[17,18]$. Definindo uma perturbação espacialmente não-homogênea de pequena amplitude deste equilíbrio, $\mathbf{u}=\mathbf{U}-\mathbf{U}_{\infty}=\left(u^{K}, u^{L}\right)^{\top}$, podemos linearizar (4a)-(4c) em torno de $\mathbf{U}_{\infty}$. Usando o Teorema de Taylor e desconsiderando os termos de maior ordem obtemos:

$$
\frac{\partial \mathbf{u}}{\partial t}+\mathbf{D}_{\chi} \frac{\partial^{2} \mathbf{u}}{\partial x^{2}}+\mathbf{D}_{\rho} \frac{\partial \mathbf{u}}{\partial x}=\mathbf{A} \mathbf{u}
$$

onde:

$$
\mathbf{A}=\left.\left(\begin{array}{cc}
\frac{\partial h}{\partial K} & \frac{\partial h}{\partial L} \\
\frac{\partial g}{\partial K} & \frac{\partial g}{\partial L}
\end{array}\right)\right|_{\mathbf{U}_{\infty}}=\left(\begin{array}{cc}
-\beta(1-\phi) & \beta(1-\phi) \\
0 & -1
\end{array}\right)
$$

é a matriz Jacobiana de $\mathbf{f}$ calculada no ponto $\mathbf{U}_{\infty}, \mathbf{D}_{\chi}=\left(\begin{array}{cc}-1 & 0 \\ \chi & -d\end{array}\right)$ e $\mathbf{D}_{\rho}=\left(\begin{array}{cc}-\rho & 0 \\ 0 & 0\end{array}\right)$. Aplicando a transformada espacial de Fourier em (7), obtemos o seguinte sistema de primeira ordem dependente do tempo:

$$
\partial_{t} \tilde{\mathbf{u}}-k^{2} \mathbf{D}_{\chi} \tilde{\mathbf{u}}+i k \mathbf{D}_{\rho} \tilde{\mathbf{u}}=\mathbf{A} \tilde{\mathbf{u}}
$$


onde $\tilde{\mathbf{u}}(t, k)$ é a transformada de Fourier de $\mathbf{u}(t, x)$. Assim, a perturbação pode ser representada como a soma de ondas harmônicas espaciais $e^{-i k x} \tilde{\mathbf{u}}(t, k)$ :

$$
\mathbf{u}(t, x)=\frac{1}{2 \pi} \int_{-\infty}^{\infty} e^{-i k x} \tilde{\mathbf{u}}(t, k) d k
$$

onde $\tilde{\mathbf{u}}(t, k)$ é solução de $(9)$, isto é:

$$
\partial_{t} \tilde{\mathbf{u}}=\left(\mathbf{A}+k^{2} \mathbf{D}_{\chi}-i k \mathbf{D}_{\rho}\right) \tilde{\mathbf{u}} .
$$

Podemos resolver a equação (11) usando o método de Euler envolvendo os autovalores da matriz $\left(\mathbf{A}+k^{2} \mathbf{D}_{\chi}-i k \mathbf{D}_{\rho}\right)$ para cada número de onda $k$. Procurando soluções exponenciais $e^{\sigma t} \mathbf{v}$ para (11), obtemos o problema de autovalor $\sigma \mathbf{v}=\left(\mathbf{A}+k^{2} \mathbf{D}_{\chi}-i k \mathbf{D}_{\rho}\right) \mathbf{v}$, ou, equivalentemente, precisamos encontrar soluções não-triviais $\mathbf{v}$ para o sistema:

$$
\mathbf{M v}=\left(\sigma \mathbf{I}-\mathbf{A}-k^{2} \mathbf{D}_{\chi}+i k \mathbf{D}_{\rho}\right) \mathbf{v}=\mathbf{0},
$$

o qual pode ser escrito como:

$$
\left(\begin{array}{cc}
\left(\sigma+\beta(1-\phi)+k^{2}-i \rho k\right) & -\beta(1-\phi) \\
-\chi k^{2} & \left(\sigma+d k^{2}+1\right)
\end{array}\right)\left(\begin{array}{c}
C \\
D
\end{array}\right)=\left(\begin{array}{l}
0 \\
0
\end{array}\right)
$$

onde $i=\sqrt{-1}$ e $\sigma(k)$ é a taxa de crescimento do modo com número de onda $k$ e amplitude $\mathbf{v}=(C, D)^{\top}$. O sistema linear (12) admite solução não-trivial se e somente se $\mathbf{M}$ é singular, isto é, se:

$$
P(\sigma)=\operatorname{det} \mathbf{M}=\left(\sigma+\beta(1-\phi)+k^{2}-i k \rho\right)\left(\sigma+d k^{2}+1\right)-\beta(1-\phi) \chi k^{2}=0 .
$$

Esta equação característica pode convenientemente ser reescrita como:

$$
P(\sigma)=\sigma^{2}+\hat{z} \sigma+\hat{w}=0
$$

onde:

$$
\begin{aligned}
& \hat{z}=z+i b=\left[(1+d) k^{2}+\beta(1-\phi)+1\right]+i(-\rho k) \\
& \left.\hat{w}=w+i d=\left\{d k^{4}+[\beta(1-\phi)(d-\chi)+1] k^{2}+\alpha \beta(1-\phi)\right]\right\}+i\left[-\rho k\left(d k^{2}+1\right)\right]
\end{aligned}
$$

Observe que o parâmetro $\rho$, envolvendo o custo de transporte, aparece apenas nas partes imaginárias de $\hat{z}$ e $\hat{w}$. A seguir iremos utilizar a seguinte definição em nossa análise: o equilíbrio espacialmente homogêneo $\mathbf{U}_{\infty}$ é (linearmente) estável se $\operatorname{Re}\{\sigma\}<0$, i.e., se todos os modos vão para zero quando $t \rightarrow \infty$.

Proposição 2 - Para que o ponto de equilíbrio $\mathbf{U}_{\infty}$ seja linearmente instável é necessário que a seguinte relação de dispersão seja satisfeita:

$$
d(d-z b)>z^{2} w
$$

onde $b, d, z$ e $w$ são dados em (15a)-(15b).

Prova: As duas raízes de (14) são dadas por:

$$
\sigma_{1}=\frac{1}{2}\left(-\hat{z}+\sqrt{\hat{z}^{2}-4 \hat{w}}\right) \text { e } \sigma_{2}=\frac{1}{2}\left(-\hat{z}-\sqrt{\hat{z}^{2}-4 \hat{w}}\right) .
$$

cujas partes reais são:

$$
\operatorname{Re}\left\{\sigma_{1}\right\}=\frac{1}{2}\left(-z+\sqrt{\frac{\sqrt{e^{2}+f^{2}}+e}{2}}\right) \text { e } \operatorname{Re}\left\{\sigma_{2}\right\}=\frac{1}{2}\left(-z-\sqrt{\frac{\sqrt{e^{2}+f^{2}}+e}{2}}\right),
$$


sendo $e=z^{2}-b^{2}-4 w$ e $f=2 z b-4 d$. Como $\operatorname{Re}\left\{\sigma_{2}\right\}$ é sempre negativa, $\mathbf{U}_{\infty}$ será instável se e somente se $\operatorname{Re}\left\{\sigma_{1}\right\}>0$. Após alguma álgebra podemos mostrar que esta condição é equivalente ao resultado desejado (16)

Se não há custo de transporte, isto é, se $\rho_{K}=0(\Leftrightarrow \rho=0)$, então $b=d=0$ e (16) se reduz a $z^{2} w<0$. Isto nos dá o seguinte corolário:

Corolário 1 - Se não há custo de transporte, ou seja, se $\rho=0$, então $\mathbf{U}_{\infty}$ é instável se e somente se $w<0$.

Neste caso particular, recuperamos o resultado obtido em $[14,15]$, o qual implica que existe um valor crítico para a intensidade de migração da mão-de-obra induzida pelo capital $\bar{\chi}$ tal que se $\chi<\bar{\chi}$, o ponto de equilíbrio $\mathbf{U}_{\infty}$ é estável, e se $\chi>\bar{\chi}, \mathbf{U}_{\infty}$ é instável. Além disto, tal valor crítico é dado por:

$$
\bar{\chi}=\left(\sqrt{\frac{1}{\beta(1-\phi)}}+\sqrt{d}\right)^{2} .
$$

Desta forma, se $\chi>\bar{\chi}$, teremos a existência de um intervalo de números de onda $\left(k_{1}, k_{2}\right)$ onde $w(k)<0$, e portanto o equilíbrio será instável. Em termos dos parâmetros originais, temos a seguinte condição para instabilidade:

$$
\chi_{L}>b\left(\frac{\delta^{\phi}}{s A}\right)^{\frac{1}{1-\phi}}\left(\sqrt{\frac{\delta d_{L}}{a}}+\sqrt{\frac{d_{K}}{1-\phi}}\right)^{2}=: \chi_{c} .
$$

Caso não haja migração de mão-de-obra induzida pelo capital, $\chi_{L}=0(\Leftrightarrow \chi=0)$, temos que (16) é equivalente à $-\rho^{2} k^{2}>z^{2}$. Neste caso, o equilíbrio é sempre estável, o que resulta no:

Corolário 2 - Se não há migração de mão-de-obra induzida pelo capital, isto é, se $\chi=0$, então $\mathbf{U}_{\infty}$ é estável. Ou seja, a existência de tal migração de mão-de-obra na economia é condição necessária para a formação de aglomerados ou ciclos econômicos, assim como encontrado em $[14,15]$.

Corolário 3 - Considere um custo de transporte não-nulo, $\rho>0$. Então, para que $\mathbf{U}_{\infty}$ seja instável é necessário, mas não suficiente, que $w<0$.

Prova - Para mostrar isto, observe que (16) pode ser reescrito como:

$$
\rho^{2}<-\frac{z^{2} w}{v}
$$

onde:

$$
\begin{aligned}
& z=(1+d) k^{2}+\beta(1-\phi)+1 \\
& w=d k^{4}+[\beta(1-\phi)(d-\chi)+1] k^{2}+\beta(1-\phi) \\
& v=d k^{6}+[\beta(1-\phi) d+1] k^{4}+\beta(1-\phi) k^{2}
\end{aligned}
$$

Como $d, \beta>0$ e $\phi \in(0,1)$, então $z, v>0$. Desta forma, para a desigualdade (19) ser satisfeita é necessário que $w<0$

Finalmente, segue diretamente de (17) e (19) o resultado principal deste trabalho: 
Proposição 3 - Considere que $\chi>\bar{\chi}$. Então, existe um valor crítico $\bar{\rho}$ tal que se $\rho<\bar{\rho}$, o ponto de equilíbrio $\mathbf{U}_{\infty}$ é instável, e se $\rho>\bar{\rho}, \mathbf{U}_{\infty}$ é estável. Além disto, este valor crítico é dado por:

$$
\bar{\rho}=\max _{k \in\left[k_{1}, k_{2}\right]} \sqrt{-\frac{z^{2} w}{v}}
$$

onde $z, w$ e $v$ são dados por (20a)-(20c). Voltando aos parâmetros originais, isso implica que o valor crítico para o custo de transporte em termos físicos é dado por $\rho_{c}=\sqrt{\frac{a}{d_{K}}} \bar{\rho}$, desde que $\chi_{L}>\chi_{c}$ (18). Assim, se $\rho_{K}<\rho_{c}$, o ponto de equilíbrio é instável, e se $\rho_{K}>\rho_{c}$, ele é estável.

Este resultado é importante pois reproduz, em um modelo de crescimento espacial macroeconômico, um resultado fundamental obtido com o Modelo de Centro-Periferia da Nova Geografia Econômica (modelos estes microeconômicos): de que existe um valor crítico para o custo de transporte acima do qual a economia se torna homogênea, ao passo que, se este é pequeno o suficiente (abaixo deste valor crítico) a economia apresenta a formação de aglomerados [10, 11]. Dada a restrição de espaço, neste trabalho não apresentaremos simulações numéricas, mas a medida que o custo de transporte vai diminuindo, as soluções do modelo apresentam os quatro tipos de dinâmica espaço-temporal apresentadas em [14, 15]: (i) a economia converge para um estado estacionário homogêneo; (ii) a economia converge para um estado estacionário nãohomogêneo, com a formação de aglomerados de capital e trabalho; (iii) a economia desenvolve ciclos periódicos; e (iv) a economia desenvolve ciclos aperiódicos ou caóticos.

Observação - A análise de estabilidade feita aqui é válida para um domínio ilimitado. Se restringirmos estes resultados ao intervalo $\Omega=[0, l]$, e considerarmos as condições de contorno (3d), obtemos autovalores dados pelos números de onda discretos, $k=\frac{n \pi}{l} \Rightarrow q=k^{2}=\frac{n^{2} \pi^{2}}{l^{2}}$, $n=0,1,2, \ldots$ e então a perturbação $\mathbf{u}(t, x)$ dada em (10) pode ser escrita como a série de Fourier de cossenos:

$$
\mathbf{u}(t, x)=\sum\left(\begin{array}{c}
C_{n} \\
D_{n}
\end{array}\right) e^{\sigma\left(k^{2}\right) t} \cos \left(\frac{n \pi x}{l}\right)
$$

onde as constantes $C_{n}$ e $D_{n}$ dependem das condições iniciais (3c). Se as hipóteses da Proposição 3 são satisfeitas, temos que $\sigma\left(k^{2}\right)$ é positivo apenas em um intervalo de números de onda $k \in\left(k_{1}, k_{2}\right)$. Aqui $k_{1}$ e $k_{2}$ são as raízes positivas da parábola (16), e depende de $\chi$ e $\rho$. Como os números de onda são discretos, temos que: $n_{1}=\left\lceil\frac{k_{1}(\chi, \rho) l}{\pi}\right\rceil$ e $n_{2}=\left\lfloor\frac{k_{2}(\chi, \rho) l}{\pi}\right\rfloor$. Desta forma podemos ver que, além de $\chi>\bar{\chi}$ e $\rho>\bar{\rho}$, o tamanho do domínio, $l$, deve ser grande o suficiente para garantir a existência de números naturais $n \in\left[n_{1}, n_{2}\right]$, e portanto a existência de modos instáveis. Chamamos este tamanho crítico que garante a existência de modos instáveis de $l_{c}=l_{c}(\chi, \rho)$. Assim, (21) pode ser aproximada como a soma apenas dos modos de amplitude crescente: $\mathbf{u}(t, x) \approx \sum_{n=n_{1}}^{n_{2}}\left(\begin{array}{c}C_{n} \\ D_{n}\end{array}\right) e^{\sigma\left(k^{2}\right) t} \cos \left(\frac{n \pi x}{l}\right)$

\section{Conclusões e Perspectivas}

Neste trabalho derivamos um valor crítico para o custo de transporte do bem agregado tal que, se o custo for menor do que este valor crítico (e se a intensidade da migração de mão-de-obra induzida pelo capital for forte o suficiente e se a economia for grande o suficiente), a economia entra em um regime de instabilidade onde pode convergir para um estado estacionário nãohomogêneo, com a formação de aglomerados de capital e trabalho; desenvolver ciclos periódicos; ou desenvolver ciclos aperiódicos ou caóticos, dependendo dos parâmetros. Desta forma reproduzimos, com um modelo macroeconômico, um resultado fundamental da Nova Geografia Econômica. A análise de estabilidade apresentada aqui pode facilmente ser estendida para uma economia bidimensional. Trabalhos futuros considerarão custos de congestionamento no modelo, além de realizar simulações numéricas uni e bidimensionais. 


\section{Referências}

[1] R. J. Barro e X. Sala-i-Martin, Economic Growth, 2nd edition, MIT Press, US, 2004.

[2] R. Boucekkine, C. Camacho e G. Fabbri, Spatial dynamics and convergence: The spatial AK mopdel, Journal of Economic Theory, 148:2719-2736, Elsevier, 2013.

[3] P. Brito, The dynamics of growth and distribution in a spatially heterogeneous world, Working Paper, ISEG, WP13/2004/DE/UECE, 2004.

[4] P. Brito, Essays in Honour of Antonio Simoes Lopes, A Spatial Solow Model with Unbounded Growth, pages 277-298, ISEG/UTL, 2005.

[5] C. Camacho e B. Zou, The spatial solow model, Economics Bulletin, 18(2):1-11, 2004.

[6] C. Camacho, B. Zou e M. Briani, On the dynamics of capital accumulation across space, European Journal of Operational Research, (186):451-465, 2008.

[7] V. Capasso, R. Engbers e D. La Torre, On a spatial solow model with tech- nological diffusion and nonconcave production function, Nonlinear Analysis: Real World Applications, (11):3858-3876, 2010.

[8] V. Capasso, R. Engbers e D. La Torre, Population dynamics in a spatial Solow model with a convex-concave production function, Mathematical and Statistical Methods for Actuarial Sciences and Finance, pages 61-68, Springer-Verlag, Milano, Italy, 2012.

[9] B. B. Edelstein, Cell specific diffusion model and morphogenesis, Journal of Theoretical Biology, 30:515-532, 1971.

[10] M. Fujita, P. Krugman e A. J. Venables, The Spatial Economy, MIT Press, US, 1999.

[11] M. Fujita e J-F Thisse, Economics of Agglomeration: Cities, Industrial Location, and Regional Growth, Cambridge University Press, UK, 2002.

[12] W. Isard, Location and space-economy: a general theory relating to industrial location, market areas, land use, trade, and urban structure, MIT Press \& Wiley, NY, 1956

[13] W. Isard e P. Liossatos, Spatial Dynamics and Optimal Space-Time Development, volume 4 of Studies in Regional and Urban Economics, North-Holland, New York, US, 1979.

[14] J. P. Juchem Neto, Modelos Evolutivos de Crescimento Econômico com Dependência Espacial, Tese de Doutorado, Instituto de Matemática, UFRGS, Porto Alegre, RS, 2013. Disponível eletronicamente no endereço: http://hdl.handle.net/10183/69937.

[15] J. P. Juchem Neto e J. C. R. Claeyssen, Capital-induced labor migration in a spatial solow model, XVI Encontro de Economia da Região Sul (Anpec-Sul), Curitiba, PR, 2013.

[16] J. M. Quigley, The renaissance in regional research, The Annals of Regional Sciences, Springer-Verlag, 35:167-178, 2001.

[17] E. F. Keller e L. A. Segel, Initiation of slime mold aggregation viewed as an instability, Journal of Theoretical Biology, Volume 26, N. 3, p. 399-415. March 1970.

[18] J. D. Murray, Mathematical Biology - II: Spatial Models and Biomedical Applications, 3rd Edition, Springer-Verlag, New York, 2003. 